\title{
SELEÇÃO DE ISOLADOS DE FUNGOS ENTOMOPATOGÊNICOS PARA O CONTROLE DE LEPTOPHARSA HEVEAE (HEMIPTERA: HETEROPTERA, TINGIDAE)
}

\author{
E.A.R. Silva ${ }^{1}$, A. Batista Filho' ${ }^{1}$, I.M. Wenzel ${ }^{1}$, E.L. Furtado², J.E.M. Almeida ${ }^{1}$ \\ ${ }^{1}$ Instituto Biológico, Centro Experimental Central, CP 70, CEP 13012-970, Campinas, SP, Brasil. E-mail: \\ batistaf@biologico.sp.gov.br
}

\section{RESUMO}

Foram analisados vinte e sete isolados de fungos entomopatogênicos procedentes de diversos hospedeiros e regiões. A produção de conídios dos patógenos foi efetuada em placas de Petri contendo meio de cultura BDA. Insetos de terceiro instar de Leptopharsa heveae foram inoculados com uma suspensão fúngica na concentração de $1 \times 10^{9}$ conídios $/ \mathrm{mL}$. Avaliações de mortalidade confirmada foram efetuadas a cada dois dias e evidenciaram grande variação de virulência, sendo o isolado 1.189, de Metarhizium anisopliae, o mais virulento. Os cinco melhores isolados, mais os dois tidos como padrão por empresas do setor de heveicultura, foram então submetidos aos testes de produção. Para tanto, pré-matrizes em placas de Petri contendo meio de cultura BDA foram preparadas. A partir destas placas, matrizes contendo arroz como meio de cultura foram inoculadas com suspensão de conídios originária das pré-matrizes na concentração de $5 \times 10^{7}$ conídios / $\mathrm{mL}$. Concluído o desenvolvimento dos patógenos nas matrizes, sacos de polipropileno contendo canjica de milho como meio de cultura foram inoculados com $40 \mathrm{~mL}$ da suspensão obtida a partir das matrizes. Após 15 dias de incubação, o conteúdo das sacolas foi avaliado quanto à produção e viabilidade de conídios. O isolado 1200, de Isaria fumosorosea, apresentou os melhores resultados de rendimento total e taxa de viabilidade e o isolado E9 de Metarhizium anisopliae foi o mais virulento ao percevejo de renda.

PALAVRAS-CHAVE: Controle microbiano, percevejo de renda, seringueira, produção total, eficiência patogênica.

\begin{abstract}
SELECTION OF ENTOMOPATHOGENIC FUNGI STRAINS TO LEPTOPHARSA HEVEAE (HEMIPTERA: TINGIDAE) CONTROL. Twenty-seven strains of entomopathogenic fungi from diverse hosts and geographic regions were tested for their virulence, total conidia production and viability rate. The conidia production was made in PDA medium. Leptopharsa heveae third instar nymphs were inoculated with fungi suspension at the concentration of $1 \times 10^{9}$ conidia $/ \mathrm{mL}$. In each two days, confirmed mortality evaluations were done and a large variation of virulence was observed, been 1,189, of Metarhizium anisopliae, the best one. The top five strains, plus the two standards, were then submitted to production tests. For this purpose, pre-matrices in PDA medium were prepared, from which matrices in rice medium were after inoculated at the concentration of $5 \times 10^{7}$ conidia/mL. From the rice matrices, polypropylene bags with maize medium were inoculated with $40 \mathrm{~mL}$ of these suspensions. After fifteen days of incubation, the bags content were evaluated for total conidia production and their viability. The 1200 strain, of Isaria fumosorosea, presented the best results in total yield and viability rate; to E9 strain of de Metarhizium anisopliae was more virulent to rubber tree lacebug.
\end{abstract}

KEY WORDS: Microbial control, rubber tree lacebug, rubber tree, production, pathogenic efficiency.

\section{INTRODUÇÃO}

A seringueira (Hevea brasiliensis) pertence à família Euphorbiaceae e tem como habitat natural a região amazônica brasileira (GASPAROTTO et al., 1997), cons- tituindo-se na principal fonte de borracha natural produzida no mundo.

Segundo estimativas do International Rubber Study Group (IRSG), em 2004, no Brasil, para um consumo de 260 mil toneladas, foram importadas

${ }^{2}$ Universidade Estadual Paulista, Faculdade de Ciências Agrárias. Botucatu, SP, Brasil. 
160 mil toneladas de borracha natural. Nesse mesmo ano, o país atingiu a produção recorde de $100 \mathrm{mil}$ toneladas (www.revistaanalytica.com.br/analytica/ ed_anteriores/26/art01.pdf).

A Associação Paulista de Produtores e Beneficiadores de Borracha (Apabor) estima que, em 2030, a demanda brasileira deva alcançar 1 milhão de toneladas (CORTEZ, 2005).

Diversas tentativas foram realizadas para tornar o país autossuficiente nessa matéria-prima, como o Programa de Incentivo à Produção de Borracha Natural (Probor I, II, III). Porém, o aparecimento de doenças e pragas nos seringais tem tornado muito difícil a implantação destas florestas.

Dentre as pragas que atingem a referida cultura está a espécie Leptopharsa heveae (Hemiptera: Tingidae), popularmente conhecida como percevejo de-renda ou mosca-de-renda. Esta praga vem merecendo destaque devido à sua ampla distribuição geográfica e alta capacidade reprodutiva, mas, principalmente, em função dos prejuízos por ela causados. Seringueiras atingidas por L. heveae têm a produção de látex reduzida e fenologia alterada, o que provoca a troca precoce das folhas, num período que favorece o ataque do fungo Microcyclus ulei (WILCKen et al., 1995).

A convivência com esta praga é, no entanto, inevitável, pois se encontra distribuída em grande parte do território nacional. $\mathrm{O}$ manejo integrado de pragas (MIP) é uma alternativa que, além de favorecer várias opções para o controle de pragas, as mantém em níveis baixos, mesmo com a diminuição do uso de defensivos químicos (PAPECEK; SMITH, 1994).

Políticas internacionais demandam fortemente alternativas para os agrotóxicos, e a utilização de entomopatógenos contra pragas é uma alternativa promissora. No entanto, a eficiência de um entomoparasita no controle de uma praga é influenciada tanto por fatores bióticos como abióticos. Entre os fatores bióticos, ALVES (1998) relatou que, para haver uma epizootia, é necessário que a população da praga esteja elevada para favorecer a disseminação do parasita na plantação; haja dispersão do inseto contaminado na área; o patógeno apresente alta virulência, alta capacidade de reprodução e capacidade de sobrevivência no ambiente.

Osfungosentomopatogênicos Sporothrixinsectorum, Metarhizium anisopliae, Beauveria bassiana Isaria spp. vêm sendo amplamente estudados e utilizados no controle microbiano de pragas, sendo importante comentar as recentes discussões quanto à taxonomia de"S. insectorum", pois estudos moleculares indicam que isolados do fungo identificado como "Sporothrix insectorum" deHoog \& H. C. Evans pertencem a mais de uma espécie e não são espécies verdadeiras de Sporothrix (MicherefF FilHo et al., 2007). Contudo, para facilitar o entendimento no trabalho, manteremos a nomenclatura "S. insectorum" neste estudo, deixando claro que os isolados avaliados podem ter outra classificação.

Alguns autores estudaram a eficiência de controle deste percevejo com uso de $S$. insectorum, dentre eles destacamos TANZINI (2002) e Alves et al. (2003).

Diante do exposto, o trabalho teve como objetivo avaliar e comparar a eficiência dos fungos "S. insectorum", M. anisopliae, B. bassiana, e Isaria spp. no controle de Leptopharsa heveae através de seleção de isolados em laboratório, levando-se em consideração não só a virulência e o potencial de produção em larga escala, mas também a viabilidade desta produção (propágulos viáveis).

\section{MATERIAL E MÉTODOS}

Nos bioensaios foram utilizados isolados de $B$. bassiana (Bb), M. anisopliae (Ma), Isaria fumosorosea (Ifu), Isaria farinosa (Ifa) e "S. insectorum" (Si) provenientes da Micoteca de fungos do Laboratório Agrobiológico das Plantações E. Michelin Ltda. e da Coleção de Micro-organismos Entomopatogênicos "Oldemar Cardim Abreu" do Laboratório de Controle Biológico do Instituto Biológico de São Paulo. Foram testados 27 isolados procedentes de diversos hospedeiros e regiões (Tabela 1).

Todos os isolados foram previamente revigorados a partir do próprio inseto. A produção de conídios dos patógenos foi efetuada em placas de Petri com meio de cultura BDA (200 g de batata, $20 \mathrm{~g}$ de dextrose, $15 \mathrm{~g}$ de ágar e $1.000 \mathrm{~mL}$ de água), em câmara climatizada a $26 \pm 0,5^{\circ} \mathrm{C}$ e fotofase de 12 horas. Após dez a doze dias de incubação, os conídios foram colhidos com espátula para o preparo do inóculo.

Para uso nos ensaios, insetos de $3^{\circ}$ instar do percevejo foram coletados no seringal das Plantações E. Michelin Ltda., em Itiquira, MT. O bioensaio foi constituído de 28 tratamentos, sendo 27 isolados e 1 testemunha, e contou com 5 repetições; onde cada uma delas continha 10 insetos/placa de Petri.

Cada placa teve a superfície interna revestida por papel filtro umedecido e folhas de seringueira adicionadas para alimentação dos insetos. Dez insetos por placa foram colocados com o auxilio de um pincel $\mathrm{n}^{\circ} 0$, visando minimizar os danos mecânicos a eles. Em seguida, realizou-se a inoculação destes insetos nas placas com suspensão do patógeno na concentração de $1 \times 10^{9}$ conídios/mL e volume de 1 $\mathrm{mL}$ por placa. Fez-se uso nesta etapa de um aparelho borrifador ajustado para ejetar $1 \mathrm{~mL}$ de spray a cada acionamento. Como o critério de seleção deste estudo foi baseado essencialmente em virulência, e não em concentração, adotamos $10^{9}$ conídios $/ \mathrm{mL}$, por suas maiores possibilidades de eficiência. 
Tabela 1 - Isolados de fungos entomopatogênicos utilizados no estudo de controle do percevejo de renda da seringueira Leptopharsa hevea.

\begin{tabular}{|c|c|c|c|}
\hline Isolados & Patógenos & Procedência & Hospedeiro \\
\hline 969 & Beauveria bassiana & Piracicaba, SP & Blatella germanica \\
\hline 1196 & Beauveria bassiana & Corumbá, MS & Solo \\
\hline 307 & Beauveria bassiana & Araras, SP & Diatrea saccharalis \\
\hline PL 63 & Beauveria bassiana & Guairá, SP & Atta sp. \\
\hline 447 & Beauveria bassiana & Cuiabá, MT & Solenopsis invicta \\
\hline IBCB66 & Beauveria bassiana & S. J. do Rio Pardo, SP & Hypothenemus hampei \\
\hline IBCB87 & Beauveria bassiana & Goiânia, GO & Cosmopolites sp. \\
\hline IBCB330 & Beauveria bassiana & Guaraniaçu, PR & Mata nativa \\
\hline E9 & Metarhizium anisopliae & Boca da Mata, AL & Mahanarva posticata \\
\hline E6 & Metarhizium anisopliae & Pernambuco & Diatrea saccharalis \\
\hline 1149 & Metarhizium anisopliae & & \\
\hline RJC & Metarhizium anisopliae & Desconhecido & Deconhecido \\
\hline 1189 & Metarhizium anisopliae & Corumbá, MS & Solo \\
\hline 1175 & Metarhizium anisopliae & Córrego Rico, SP & Solo \\
\hline IBCB425 & Metarhizium anisopliae & Iporanga, $\mathrm{SP}$ & Lagarta \\
\hline 1200 & Isaria fumosorosea & Amélio Rodrigues, BA & Mahanarva posticata \\
\hline IBCB148 & Isaria fumosorosea & Limeira, SP & Solo \\
\hline IBCB124 & Isaria fumosorosea & Campinas, SP & Solo \\
\hline 1205 & Isaria farinosa & Santa Fé do Sul, SP & Bemisia tabaco \\
\hline IBCB108 & Isaria farinosa & Cosmópolis, SP & Solo \\
\hline SJ1S & Sporothrix insectorum & & \\
\hline CPAC5 & Sporothrix insectorum & & \\
\hline SJRC & Sporothrix insectorum & & \\
\hline IBCB79 & Sporothrix insectorum & Itiquira, MT & Leptopharsa heveae \\
\hline IBCB88 & Sporothrix insectorum & Curitiba, PR & Leptopharsa heveae \\
\hline 1225 & Sporothrix insectorum & S. J. do Rio Claro, MT & Leptopharsa heveae \\
\hline 1226 & Sporothrix insectorum & Itiquira, MT & Leptopharsa heveae \\
\hline
\end{tabular}

Os recipientes com os insetos foram então vedados e mantidos em câmara climatizada a $26 \pm$ $2^{\circ} \mathrm{C}$ e fotofase de 12 horas durante todo o período experimental. Para o tratamento testemunha, o mesmo procedimento foi efetuado, substituindo-se a suspensão fúngica por água destilada.

As avaliações foram realizadas a cada dois dias, registrando-se o número de insetos vivos e mortos. Para confirmação da mortalidade pelo patógeno, os insetos mortos foram lavados em álcool $70 \%$ por 10 segundos e enxaguados em água destilada por 20 segundos para descontaminação externa. Em seguida, foram acondicionados em placas de Petri com câmara úmida por 15 dias, tornando possível a visualização da extrusão e reprodução do patógeno (confirmação da morte), ou não.

Os cadáveres foram observados e aqueles que confirmaram morte pelo fungo foram anotados, perfazendo os dados de mortalidade confirmada. Os cinco isolados que apresentaram mortalidade confirmada superior a $80 \%$ até o sexto dia após inoculação foram selecionados para a etapa seguinte do trabalho, além dos dois isolados considerados padrão por empresas do ramo:SJRC ("S. insectorum") e 1200 (I. fumosorosea).
Foram calculados os dados de mortalidade confirmada e mortalidade total (mortalidadeindependente da causa) até que a testemunha atingisse $100 \%$ de mortalidade.

Com base nos resultados de virulência obtidos, foram realizados testes de produção de conídios, objetivando a identificação daqueles que, além de mais virulentos em laboratório, fossem capazes de produzir os maiores números de conídios dentro de um prazo pré-estabelecido.

Neste estudo, cada isolado representou um tratamento, totalizando 7 tratamentos compostos por 5 repetições. Os isolados PL63, IBCB87, SJRC, 1225,1189 , E9 e 1200 foram repicados em placas de Petri contendo meio BDA. As placas, chamadas de pré-matrizes, foram incubadas durante dez dias em câmara climatizada a $26 \pm 2^{\circ} \mathrm{C}$ e fotofase de 12 horas para promover o desenvolvimento dos fungos. Após dez dias, os conídios foram retirados com o auxílio de alça metálica e suspensão fúngica na concentração de $5 \times 10^{7}$ conídios/mL foi preparada para inoculação das matrizes.

Para as matrizes utilizou-se arroz como meio de cultura, sendo este colocado na quantidade de $100 \mathrm{~g}$ em frasco Erlenmeyer de $1.000 \mathrm{~mL}$ acrescido de $50 \mathrm{~mL}$ 
deágua destilada eácidocítrico, na concentração de 0,3 $\mathrm{gL}^{-1}$. O frasco foi lacrado com papel alumínio e levado à autoclave por 30 minutos a temperatura de $120^{\circ} \mathrm{C}$.

Em câmara de fluxo laminar, procedeu-se à inoculação dos frascos Erlenmeyers (matrizes) adicionando-se $20 \mathrm{~mL}$ da suspensão de conídios. Estes foram então lacrados com papel alumínio e fita crepe para serem agitados de modo a possibilitar uma boa distribuição do inóculo. Prontas as matrizes, estas permaneceram em sala de germinação climatizada a $26 \pm 2^{\circ} \mathrm{C}$ e fotofase de 12 horas por 12 dias.

A partir das matrizes foram produzidas as sacolas, que correspondem à forma como estes fungos são produzidos em escala industrial. Em câmara de fluxo laminar, as matrizes foram raspadas com o auxílio de espátula de metal esterilizada, e seu conteúdo transferido para Becker de $2.000 \mathrm{~mL}$ contendo 750 $\mathrm{mL}$ de água esterilizada. A suspensão resultante foi passada por peneira de $0,84 \mathrm{~mm}$ e acrescida de uma cápsula de tetraciclina.

No preparo das sacolas, fez-se uso de 5 sacos plásticos de polipropileno para cada tratamento, nas dimensões de $30 \times 15 \mathrm{~cm}$, contendo $400 \mathrm{~g}$ de canjica de milho e $200 \mathrm{~mL}$ de solução aquosa de ácido cítrico a $0,3 \mathrm{gL}^{-1}$. As sacolas foram lacradas com grampos metálicos e autoclavadas por 40 minutos a $120^{\circ} \mathrm{C}$. Após esfriarem, foram inoculadas com $40 \mathrm{~mL}$ da suspensão obtida a partir das matrizes, utilizandose para tanto uma seringa de vidro de $20 \mathrm{~mL}$. As sacolas foram identificadas e transferidas para sala de germinação climatizada a $26 \pm 2{ }^{\circ} \mathrm{C}$ e fotofase de 12 horas por 15 dias. Ressalta-se aqui que todas as etapas de produção foram realizadas de forma intercalada, permitindo que as avaliações respeitassem rigorosamente o período de incubação de 15 dias para cada tratamento.

Para verificação da concentração (produção total) após o período de incubação de 15 dias, o conteúdo de cada sacola, separadamente, foi diluído em 1.000 $\mathrm{mL}$ de água destilada, homogeneizado e peneirado. À esta suspensão foi adicionado espalhante adesivo Tween a $0,1 \%$ e o número de conídios quantificado em câmara de Neubauer.

Para leitura da viabilidade, foram preparadas três lâminas recobertas com meio de cultura BDA por tratamento, tendo elas sido previamente marcadas com um círculo na face inferior. Sobre o meio de cultura foi inoculado, na área delimitada, 0,5 $\mathrm{mL}$ de suspensão fúngica (a mesma utilizada para análise de concentração) com $10^{5}$ conídios $/ \mathrm{mL}$. Estas lâminas, acondicionadas individualmente em placa de Petri com algodão umedecido e esterilizadas, permaneceram incubadas na ausência de luz por 15 horas em câmara climatizada a $26 \pm 2^{\circ} \mathrm{C}$. Após este período, foi colocada uma gota do corante azul de Arman sobre a área do círculo para deter a germinação e facilitar a visualização dos conídios. Foram quantificados 100 conídios na área pré-delimitada das lâminas em microscópio óptico com aumento de 400 vezes. Anotou-se o número de germinados e não germinados para posterior definição da porcentagem de viabilidade.

Os dados foram processados no programa computacional SAEG. Para determinar as diferenças significativas entre tratamentos empregou-se o teste de agrupamento Scott-Knott ou o teste de Tukey a $5 \%$ de probabilidade.

\section{RESULTADOS E DISCUSSÃO}

Ocorreu grande variação de virulência entre os isolados testados. Foram considerados eficientes nesta etapa de seleção os isolados que atingiram porcentual de mortalidade confirmada superior a $80 \%$ até o sexto dia após aplicação da suspensão fúngica (Tabela 2). O teste de agrupamento Scott-Knott confirmou a seleção realizada, porém, evidenciou que em algumas situações a mortalidade em vários tratamentos foi inferior à da testemunha, o que pode serjustificado pela sensibilidade do inseto ao estresse ocasionado pelo manuseio, mas que não interfere nos resultados, uma vez que eles foram baseados em valores de mortalidade confirmada.

B. bassiana e M. anisopliae foram os fungos mais virulentos sobre $L$. heveae, apresentando maior número de isolados selecionados, enquanto " $\mathrm{S}$. insectorum" mostrou-se mediano, com apenas 1 representante (Tabela 2). TANZINI (2002) observou valores de mortalidade confirmada de L. heveae variando entre $25 \%$ e $90 \%$ quando inoculados por isolados de $S$. insectorum. Em experimentos de laboratório, SCOMPARIN (2000) relatou eficiência de controle de $100 \%$ de S. insectorum sobre L. heveae após oito dias de pulverização de suspensão fúngica com concentrações entre $1 \times 10^{6} \mathrm{e} 1 \times 10^{9}$ conídios $/ \mathrm{mL}$.

Isaria sp. pode ser considerado o fungo de pior desempenho, uma vez que sua máxima eficiência foi conferida pelo isolado IBCB 124, que atingiu mortalidade confirmada de $80 \%$ ao sexto dia após aplicação da suspensão fúngica, seguido pelo isolado 1200 de I. fumosorosea, com 74\%. TANZINI (2002), em experimento semelhante, observou valores de $80 \%$ de mortalidade confirmada para L. heveae quando inoculado com o mesmo isolado. Quanto à Isaria sp., resultados semelhantes foram observados por Wraight et al. (1998), que testaram isolados de Isaria spp. para ocontroledamosca-branca, Bemisiaargentifolii. Em casa de vegetação, I. fumosorosea causou $70 \%$ de mortalidade em B. argentifolii (VIDAL et al., 1998).

Atualmente, algumas empresas vêm praticando o controle microbiano contra $L$. heveae através da utilização dos fungos "S. insectorum" e Isaria sp., produzidos segundo a mesma metodologia deste 
estudo. Estas empresas multiplicam industrialmente os isolados SJRC e 1200, pulverizando-os em suas plantações e obtendo sucesso parcial (eficiente em épocas de alta umidade relativa do ar) no controle da praga (comunicação pessoal). Embora estes dois isolados tenham sido considerados insatisfatórios na seleção inicial de virulência, a sua inclusão na etapa seguinte do trabalho foi importante para confirmação dos testes de laboratório e maior embasamento na eventualidade de recomendação de substituição deles para uso por empresas do setor. Dessa forma, os isolados selecionados 1200 de I. fumosorosea, SJRCe

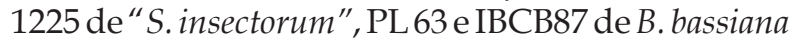
e1189 eE9 deM. anisopliae foram avaliados nas etapas de rendimento na produção em meio de cultivo.
A Figura 1 apresenta os resultados de produção média de conídios/mL obtidos após diluição do substrato sólido em $1.000 \mathrm{~mL}$ de água. Verifica-se que o isolado 1200 de I. fumosorosea apresentou maior produção de conídios (13,92 x 1077conídios/ $\mathrm{mL}$ ) quando comparado aos demais. Em seguida, os isolados PL63 de B. bassiana com 9,5 x 107conídios/ $\mathrm{mL}, 1225$ de "S. insectorum" com $7 \times 10^{7}$ conídios/ mL e IBCB87 de B. bassiana com 5,67 x 107conídios/ $\mathrm{mL}$. O pior desempenho foi do isolado 1189 de $M$. anisopliae, com produção de 1,92 ×107 conídios $/ \mathrm{mL}$. Os resultados de produtividade média de conídios confirmaram a superioridade do isolado 1200, que apresentou maior produção, diferindo-se estatisticamente dos demais (Tabela 3).

Tabela 2 - Porcentagem média de mortalidade de Leptopharsa hevea aos 2, 4 e 6 dias após inoculação das suspensões fúngicas (Temperatura $=26 \pm 2^{\circ} \mathrm{C}$ e fotoperíodo de 12 horas).

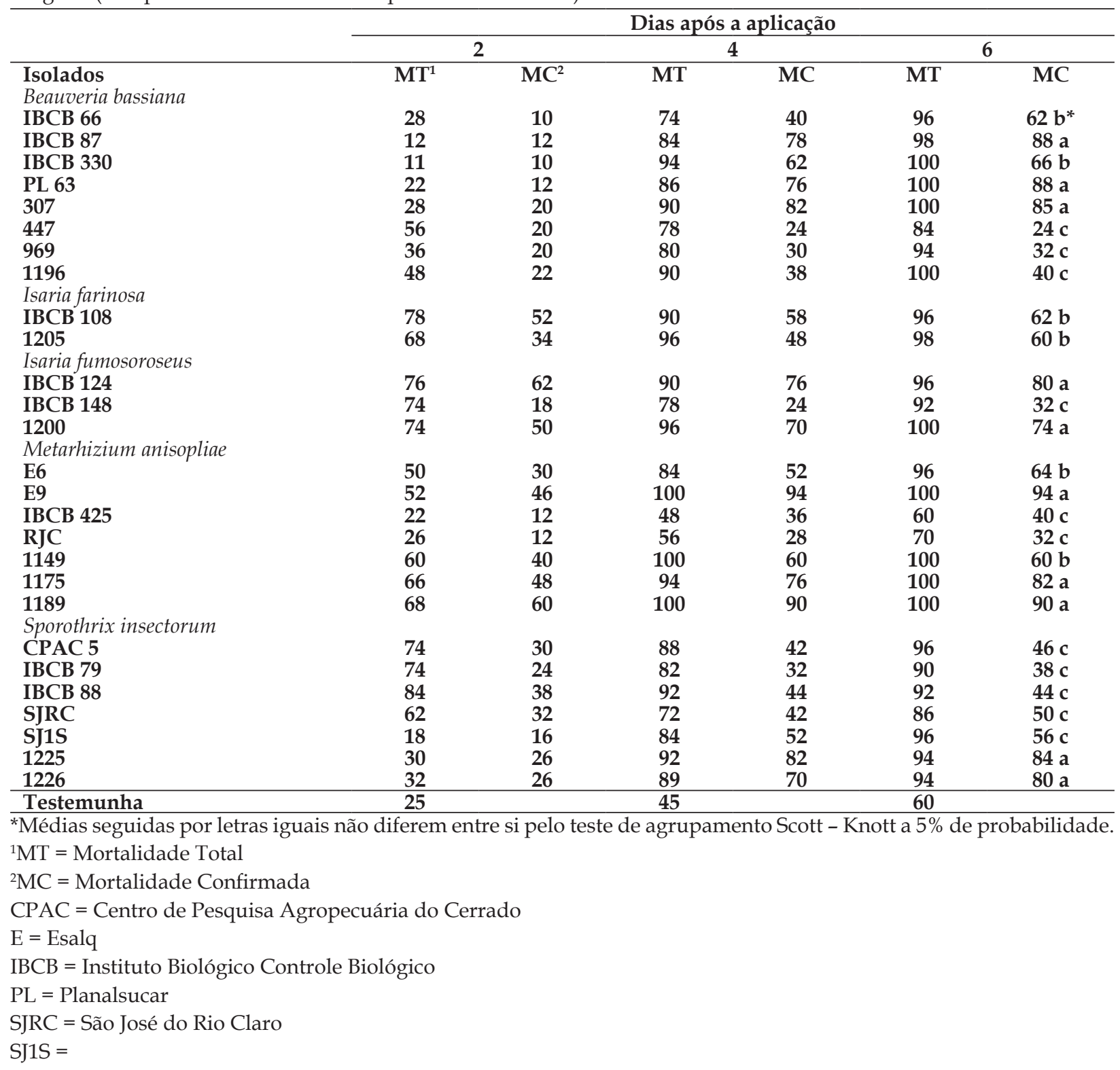


Tabela 3 - Número total de conídios/mL obtidos em cultivo em meio de canjica de milho e ácido cítrico após 15 dias de incubação (Temperatura $=26 \pm 2^{\circ} \mathrm{C}$ e fotoperíodo de 12 horas).

\begin{tabular}{lc}
\hline Tratamentos & $\begin{array}{c}\text { № de conídios } \\
\text { produzidos } / \mathrm{mL}\left(10^{7}\right)^{*}\end{array}$ \\
\hline 1200 (Isaria fumosorosea) & $13,91 \mathrm{a}$ \\
PL 63 (Metarhizium anisopliae) & $9,50 \mathrm{ab}$ \\
1225 (Sporothrix insectorum) & $7,00 \mathrm{ab}$ \\
IBCB 87 (Beauveria bassiana) & $5,66 \mathrm{ab}$ \\
E9 (Metarhizium anisopliae) & $2,67 \mathrm{~b}$ \\
SJRC (Sporothrix insectorum) & $2,17 \mathrm{~b}$ \\
1189 (Metarhizium anisopliae) & $1,97 \mathrm{~b}$
\end{tabular}

*Médias seguidas por letras iguais não diferem entre si pelo teste de Tukey, a 5\% de probabilidade.

$\mathrm{E}=$ Esalq

IBCB = Instituto Biológico Controle Biológico

$\mathrm{PL}=$ Planalsucar

SJRC = São José do Rio Claro

A produtividade de conídios para estes fungos já foi estudada por outros pesquisadores, com resultados semelhantes, variando em decorrência de metodologias distintas entre os trabalhos. Neves; Hirose (2005), em estudo de seleção de isolados de B. bassiana para o controle da broca do café, obtiveram produções de conídios/ $\mathrm{mL}$ em torno de 2,5 x $10^{6}$, enquanto Loureiro et al. (2005), estudando a produção de conídios de diversos isolados de $M$. anisopliae, observaram variações de $1,75 \times 10^{8}$ a 2,30 x $10^{8}$ conídios/g de arroz pré-cozido pelo método da bandeja, e com viabilidade média superior a $90 \%$. Bastante semelhante a este estudo também foram os resultados encontrados por LouREIRO et al. (2002), que observaram para $S$. insectorum produção de $1,84 \times$ $10^{7}$ a $2,42 \times 10^{7}$ conídios / mL.

Oisolado PL63 de B. bassiana já havia sido um dos mais eficientes quanto à virulência à L. hevea, demonstrando agora grande potencial para uso em campo por ser também um dos mais produtivos em meio sólido (Tabela 3). O isolado E9, de M. anisopliae, que se comportou como o mais virulento, atingindo $94 \%$ de mortalidade confirmada, foi o $5^{\circ}$ mais produtivo e não diferiu estatisticamente dos dois últimos colocados, SJRC de"S. insectorum"e $1189 \mathrm{de}$ M. Anisopliae (Tabela 3). O isolado SJRC, incluído como padrão, não obteve bom desempenho nos testes anteriores de virulência, e, da mesma forma, apresentou baixo rendimento em milho. O menor número de conídios produzidos/mL foi verificado para o isolado 1189 de $M$. anisopliae, que, no entanto, havia se mostrado eficiente nos bioensaios para avaliação da virulência.

Entretanto, paraqueaboa produtividadedeumisolado possa se refletir em efetiva vantagem na produção industrial e efetivo controle de pragas, é preciso que os conídios produzidos tenham viabilidade para in-

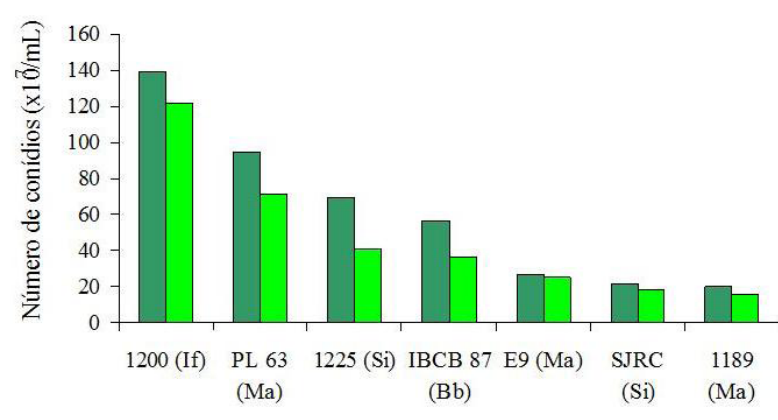

$\square$ Número de conídios produzidos $\square$ Número de conídios viáveis produzidos

Fig. 1 - Comparação entre o número total de conídios produzidos e número de conídios viáveis obtidos $/ \mathrm{mL}$ em suspensões fúngicas dos fungos Isaria fumosorosea (If), Metarhizium anisopliae (Ma), Sporothrix insectorum (Si) e Beauveria bassiana $(\mathrm{Bb})$ após 15 dias de incubação em temperatura de $26 \pm 2^{\circ} \mathrm{C}$ e fotoperíodo de 12 horas.

fectar o inseto. Observou-se que o isolado 1189 de $M$. anisopliae, o qual obteve o pior desempenho quanto à produção total deconídios (Tabela 3 ) efoi $3^{\circ}$ colocado quanto à virulência (Tabela 2), apresentou a maior porcentagem de viabilidade média de conídios entre os isolados testados, 94,3\% (Tabela 4). E9, também de M. anisopliae, tido como o segundo mais virulento na seleção inicial, atingiu 93,7\% de viabilidade média (Tabela 4). Não houve diferença significativa quanto à porcentagem de viabilidade média de conídios entre os isolados 1189 e E9, ambos de M. anisopliae. IBCB 87 , representante de $B$. bassiana, teve a pior taxa de viabilidade, estando significativamente diferenciado dos demais (Tabela 4).

É possível verificar, pelos resultados apresentados até então, que uma alta produtividade não significa vantagem quando a viabilidade é baixa e, por outro lado, a baixa produtividade pode não ser limitante, desde que haja alta porcentagem de viabilidade. Portanto, para selecionar um isolado é preciso confrontar dados de produtividade total de conídios/ $\mathrm{mL}$ com porcentagem de viabilidade.

A Figura 1 apresenta o número médio de conídios produzidos/mL e, com base nos valores de porcentagem de viabilidade, mostra o número médio de conídios viáveis produzidos pelos isolados, ou seja, a produtividade real. Observa-se que o isolado que produziu o maior número de conídios viáveis $/ \mathrm{mL}$, portanto aquele de maior produtividade real, foi o 1200 de I. fumosorosea. Embora este tratamento não seja o de maior porcentagem de viabilidade média, sua produção alcançou grande destaque entre os isolados avaliados o que resultou no maior número de conídios viáveis/mL (Tabela 5). O isolado 1189 de M. anisopliae, tido como o de maior porcentagem de viabilidade $(94,3 \%)$, atingiu apenas $1,81 \times 10^{7}$ 
Tabela 4-Viabilidade dos conídios após incubação durante 15 horas em câmara climatizada a $26 \pm 2^{\circ} \mathrm{C}$ na ausência de luz.

\begin{tabular}{lc}
\hline Tratamentos & $\begin{array}{c}\text { Viabilidade } \\
\text { média (\%)* }\end{array}$ \\
\hline 1189 (Metarhizium anisopliae) & $94,3333 \mathrm{a}$ \\
E9 (Metarhizium anisopliae) & $93,6667 \mathrm{a}$ \\
1200 (Isaria fumosorosea) & $87,0000 \mathrm{ab}$ \\
PL 63 (Metarhizium anisopliae) & $75,3333 \mathrm{abc}$ \\
SJRC (Sporothrix insectorum) & $72,0000 \mathrm{bc}$ \\
1225 (Sporothrix insectorum) & $72,0000 \mathrm{bc}$ \\
IBCB 87 (Beauveria bassiana) & $64,3333 \mathrm{c}$ \\
*Médias seguidas por letras iguais não diferem entre si \\
pelo teste de Tukey ao nível de 5\% de probabilidade. \\
E = Esalq. \\
IBCB = Instituto Biológico Controle Biológico. \\
PL = Planalsucar. \\
SJRC = São José do Rio Claro.
\end{tabular}

conídios/mL de produtividade real, não diferindo de forma significativa do último colocado para o quesito, SJRC de "S. insectorum" (Tabela 5).

Para se elevar o índice de assertividade em uma seleção de isolados realizada em condições de laboratório, é preciso avaliar o maior número de dados possível. Neste trabalho, foram avaliados fatores de virulência, produtividade total, taxa de viabilidade e produtividade real. O confronto entre quesitos mostrou que o isolado SJRC, de "Sporothrix insectorum", tido como padrão para o controle microbiano de L. heveae, obteve os piores resultados tanto quanto à virulência, como quanto à produtividade real de conídios. Isso mostra que outros isolados podem ser utilizados com resultados relevantes ao controle da praga. O isolado 1200 de I. fumosorosea, também padrão utilizado em algumas empresas do setor, alcançou virulência maior apenas em relação ao SJRC, porém, obteve a maior produtividade real entre os isolados estudados.

TANZINI (2002), durante seleção de isolados para controle da mesma praga, fez uso da concentração de 1 × $10^{8}$ conídios/mL, porém, aplicando $2 \mathrm{~mL}$ de suspensão fúngica, o dobro do volumeaqui utilizado. Outros autores, em estudos de seleção de isolados, como Cesar FilHo et al. (2002), Rohde et al. (2006) e ALMEIDAetal. (2009), testaram concentrações entre $10^{5}$ e $10^{9}$ conídios $/ \mathrm{mL}$, resultando, em geral, em proporcionalidade positiva entre concentração X eficiência.

\section{CONCLUSÃO}

O grau de virulência entre isolados é bastante variável, mesmo entre isolados de fungos de mesmo gênero.
Tabela 5 - Número total de conídios viáveis/mL obtidos em cultivo em meio de canjica de milho e ácido cítrico após 15 dias de incubação (Temperatura $=26 \pm 2^{\circ} \mathrm{C}$ e fotoperíodo de 12 horas).

\begin{tabular}{lc}
\hline Tratamentos & $\begin{array}{c}\text { No médio de conídios } \\
\text { viáveis } / \mathrm{mL}\left(10^{7}\right)^{*}\end{array}$ \\
\hline 1200 (Isaria fumosorosea) & $12,1725 \mathrm{a}$ \\
PL 63 (Metarhizium anisopliae) & $7,1025 \mathrm{ab}$ \\
1225 (Sporothrix insectorum) & $4,0467 \mathrm{ab}$ \\
IBCB 87 (Beauveria bassiana) & $3,6492 \mathrm{~b}$ \\
E9 (Metarhizium anisopliae) & $2,5358 \mathrm{~b}$ \\
1189 (Metarhizium anisopliae) & $1,8100 \mathrm{~b}$ \\
SJRC (Sporothrix insectorum) & $1,5550 \mathrm{~b}$ \\
\hline
\end{tabular}

*Médias seguidas por letras iguais não diferem entre si pelo teste de Tukey ao nível de $5 \%$ de probabilidade.

$\mathrm{E}=$ Esalq.

$\mathrm{IBCB}=$ Instituto Biológico Controle Biológico.

$\mathrm{PL}=$ Planalsucar.

SJRC = São José do Rio Claro.

O isolado E9, de M. anisopliae, é o mais virulento à $L$. heveae seguido, em ordem decrescente de virulência, por 1189 (M. anisopliae), PL63 (B. bassiana), IBCB87 (B. bassiana), 1225 (S. insectorum), 307 (B. bassiana) e 1175 (M. anisopliae).

O isolado 1200, de I. fumosorosea, apresenta os melhores resultados de produção total e viabilidade de conídios.

Não é possível estabelecer uma relação direta entre: virulência $X$ produtividade total de conídios $\mathrm{X}$ produtividade de conídios viáveis.

\section{AGRADECIMENTOS}

À Plantações E. Michelin Ltda., pela disponibilização do material necessário ao desenvolvimento desta pesquisa, bem como apoio financeiro concedido.

À Klabin S.A., para qual trabalho atualmente, por compreender a importância deste trabalho e me conceder o tempo necessário para sua conclusão.

\section{REFERÊNCIAS}

ALMEIDA, A.M.B. de; BATISTA FILHO, A.; TAVARES, F.M.; LEITE, L. G. Seleção de isolados de Beauveria bassiana para o controle de Cosmopolites sordidus (Germar, 1824) (Coleoptera: Curculonidae). Arquivos do Instituto Biológico, São Paulo, v.76, n.3, p.489-493, 2009.

CESAR FILHO. E.; MARQUES, E. J.; BARROS, R. Selection of Metarhizium anisopliae (Metsch.) and Beauveria bassiana (Bals.) isolates to control Alabama argillacea (Huebner) Caterpillars. Scientia Agricola, v.59, n.3, p.457-462, 2002. 
CORTEZ, J.V. Reflexões sobre as projeções de consumo de Borracha Natural no Brasil até o ano 2030. [Artigo 24/8/2005]. Disponível em: <http:/ /www.anba.com. br/noticia.php?id=10992>. Acesso em: 24 jun. 2006.

GASPAROTTO, L.; SANTOS, A.F.; PEREIRA, S.C.R.; FERREIRA, F.A. Doenças da seringueira no Brasil. Brasília, DF: EMBRAPA, 1997. p.10.

LOUREIRO, E.S.; BATISTA FILHO, A.; ALMEIDA J.E.M.; LEITE, L.G.; LAMAS, C. Efeito da temperatura e da luminosidade no desenvolvimento do fungo Sporothrix insectorum (Hoog \& Evans). Arquivos do Instituto Biológico, São Paulo, v.69, n.2, p.79-83, 2002.

LOUREIRO, E.S.; BATISTA FILHO, A.; ALMEIDA, J.E.M.; PESSOA, L.G.A. Produção de isolados de Metarhizium anisopliae selecionados para o controle de Mahanarva fimbriolata (STAL, 1854). Arquivos do Instituto Biológico, São Paulo, v.72, n.1, p.469-472, 2005.

MICHEREFF FILHO, M.; FARIA, M.R. de; WRAIGHT, S.P. Micoinseticidas e micoacaricidas no Brasil: Como estamos?. Brasília, DF: Embrapa Recursos Genéticos e Biotecnologia, 2007. 28p.

NEVES, P.M.O.J.; HIROSE, E. Seleção de isolados de Beauveria bassiana para o controle biológico da broca do café, Hypothenemus hampei (Ferrari) (Coleóptera: Scolytidae). Neotropical Entomology, v.34, n.1, 2005.

PAPECEK, D.; SMITH, D. Manejo integrado de pragas nos citrus: Linhas gerais de um programa comercial de MIP em Queensland, Austrália. In: SEMINÁRIO INTERNACIONAL DE CITRUS - MIP, 3., 1997. Bebedouro. Resumos. Bebedouro: 1994. p.153-175.

ROHDE, C.; ALVES, L.F.A.; NEVES, P.M.O.J.; ALVES, S.B.; SILVA, E.L. da; ALMEIDA, J.E.M. de. Seleção de Isolados de Beauveria bassiana (Bals.) Vuill. e Metarhizium anisopliae (Metsch.) Sorok. contra o Cascudinho Alphitobius diaperinus (Panzer) (Coleoptera: Tenebrionidae). Neotropical Entomology, v.35, n.2, p.231-240, 2006.

SANTOS, G.R. dos; MOTHÉ, G.G. Prospecção e perspectivas da borracha natural, Hevea brasiliensis. Revista Analytica, n.26, p.32-40, 2007. Disponível em: <http:/ / www.revistaanalytica.com.br/analytica/ed_anteriores/26/art01.pdf>. Acesso em: 9 nov. 2009.

SCOMPARIN, A.L.X. Bioecologia de percevejo-de-renda (Leptopharsa heveae Drake \& Poor, 1935) (Hemíptera: Heteroptera, Tingidae) e a avaliação do fungo entomopatogênico Sporothrix insectorum (Hoog \& Evans). 2000. 51p. Dissertação (Mestrado) - UNESP, Botucatu, SP, 2000.

TANZINI, M.R. Controle do percevejo de renda da seringueira (Leptopharsa heveae) com fungos entomopatogênicos. 2002. Tese (Doutorado em Agronomia, Área de Concentração em Entomologia Agrícola) - Escola Superior de Agricultura Luiz de Queiroz, Piracicaba, 2002.

VIDAL, C.; OSBORNE, L.S.; LACEY, L.A.; FARGUES, J. Effect of host plant on the potencial of Paecilomyces fumosoroseus (Deuteromycotina: Hyphomycetes) for controlling the silverleaf whitefly, Bemisia argentifolii (Homoptera: Aleyrodidae) in greenhouses. Biological Control, v.12, n.3. p.191-199, 1998.

WILCKEN, C.F.; FURTADO E.L.; SILVEIRA, A.P. Mosca de renda em São Paulo. 1995. 2p. (Comunicado Técnico).

WRAIGHT, S.P.; CARRUTHERS, R.I.; BRADLEY, C.A.; JARONSKI, S.T.; LACEY, L.A.; WOOD, P.; WRAIGHT, S. Pathogenicity of the entomopathogenic fungi Paecilomyces spp. and Beauveria bassiana against the silverleaf whitefly, Bemisia argentifolii. Journal of Invertebrate Pathology, v.71, n.3, p.217-226, 1998.

Recebido em 13/1/10

Aceito em 14/10/12 